\author{
Jeremy R Burt ${ }^{\star}$, Kimberly M Beavers \\ and Vincent $\mathrm{E}$ Grekoski \\ Department of Radiology, Florida Hospital Orlando, \\ USA \\ Dates: Received: 07 June, 2016; Accepted: 19 \\ August, 2016; Published: 24 August, 2016 \\ *Corresponding author: Jeremy R Burt, MD Depart- \\ ment of Radiology, Florida Hospital, 601 E Rollins \\ Street, Orlando, FL, 32803, Tel: 407-303-8178; Fax: \\ 407-303-9702l; E-mail: Jeremy.burt.md@flhosp.org \\ www.peertechz.com \\ ISSN: 2455-2976
}

\author{
Case Report
}

\section{Aortic Valve Thrombosis in Antiphospholipid Syndrome Causing Coronary Artery Embolic Disease}

\section{Abbreviations}

CMR: Cardiac MR; TTE: Transthoracic Echocardiography; RI: Magnetic Resonance Imaging; CT: Computed tomography; APS: Antiphospholipid Syndrome; SLE: Systemic Lupus Erythmatosus

\section{Introduction}

Antiphospholipid syndrome (APS) is a disorder characterized by the presence of anti-phospholipid antibodies which can include Lupus anticoagulant and Anticardiolipin antibody [1]. These antibodies bind to cardiolipin and have been shown in some cases to require $\beta_{2}$ - glycoprotein I in order to bind to cardiolipin [2]. Symptoms of this disorder include vascular thrombosis without inflammation in the vessel wall, premature birth, spontaneous abortion, and death in a morphologically normal fetus at or beyond the $10^{\text {th }}$ week of gestation [1]. APS has a strong correlation to systemic lupus erythematosus (SLE) with studies showing that $30 \%$ of patients with SLE will develop APS; however, APS can still be found in patients without SLE at a low frequency [3].

This disorder can cause cardiovascular complications which include marantic vegetation and valvular stenosis and/or regurgitation [1]. The exact mechanism of valve disease in APS is not fully understood, but the current belief is that there is an interaction between valve antigen and antiphospholipid antibodies which result in the observed thrombosis and valve thickening [4]. It is known that one third to one half of patients with APS have valve disease ${ }^{4}$ and that APS patients with valve disease have an increased risk for thromboembolic events [5].

Thromboembolic events commonly found in APS include deep vein thrombosis, stroke, pulmonary embolism, thrombosis of renal vessels, and livedo reticularis [6]. Myocardial infarction is relatively uncommon in APS patients [6] and an APS case involving infarctions due to a coronary artery embolism from aortic valve thrombosis has not been previously published. In patients without APS, valvular disease is the third most common cause of coronary embolism. The only documented cases of coronary embolism due to aortic valve vegetation were caused by prosthetic heart valve complications [7].

In this study, a patient presenting with coronary artery embolic disease due to aortic valve thrombosis and APS is documented with cardiac MR (CMR).

\section{Methods}

A 20-year-old African American male with a history of migraine, pancreatitis and ulcerative colitis presented to the ER with acute chest pain, dyspnea, and vertigo. He had no history of heart attack or stroke. The patient underwent multiple tests including laboratory testing for troponin, anticardiolipin antibodies (aCL), lupus anticoagulant (LA), and anti-beta2-glycoprotein-1 (anti-B2GP1), blood counts, renal and liver function testing. He also had an EKG, cardiac catheterization, CMR, transthoracic echocardiography, and abdominal computed tomography (CT).

\section{Results}

Laboratory tests revealed elevated troponin, thrombocytopenia, and elevated liver function tests. Electrocardiogram revealed significantST segment depression in the anterolateralleads. The patient was admitted and underwent cardiac catheterization which showed long filling defects in the large first diagonal branch (Figure 1A,B) and distal right coronary arteries (Figure $1 \mathrm{C}$ ) suspicious for embolic disease. The patient underwent transthoracic echocardiography which revealed mild mitral and tricuspid regurgitation with normal systolic function. No cardiac mass, vegetation or thrombus was identified.

Multiplanar, multisequence CMR imaging of the heart was performed before and following the intravenous administration of gadolinium to evaluate myocardial viability. The cardiac MR (Figure $2 \mathrm{~A}, \mathrm{~B}, \mathrm{~F})$ showed a $10 \times 8 \times 5 \mathrm{~mm}$ thrombus on the aortic valve in 


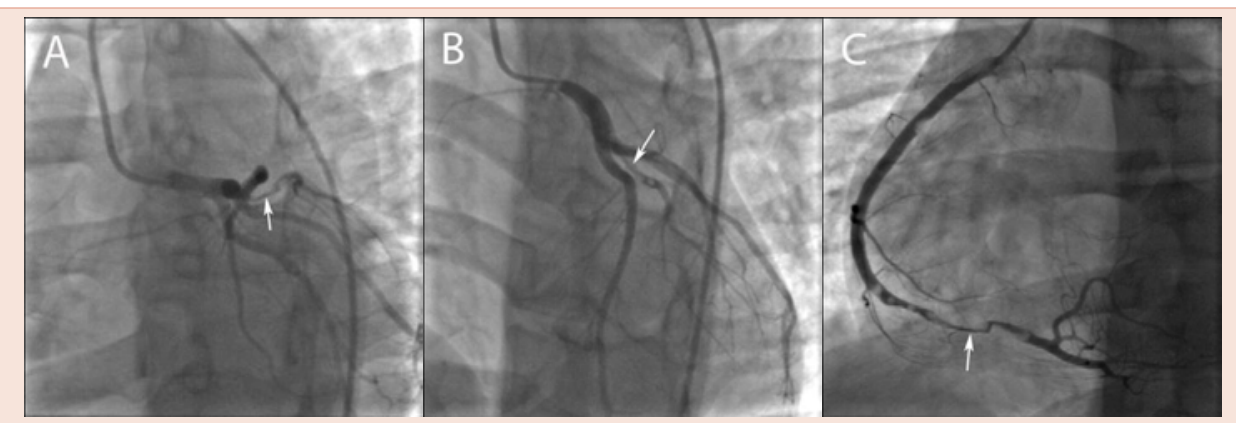

Figure 1: (A\&B) Angiogram of the left coronary artery shows string sign caused by embolus in the origin of the first diagonal branch (arrows). (C) Angiogram of the right coronary artery also shows multiple emboli (arrow).

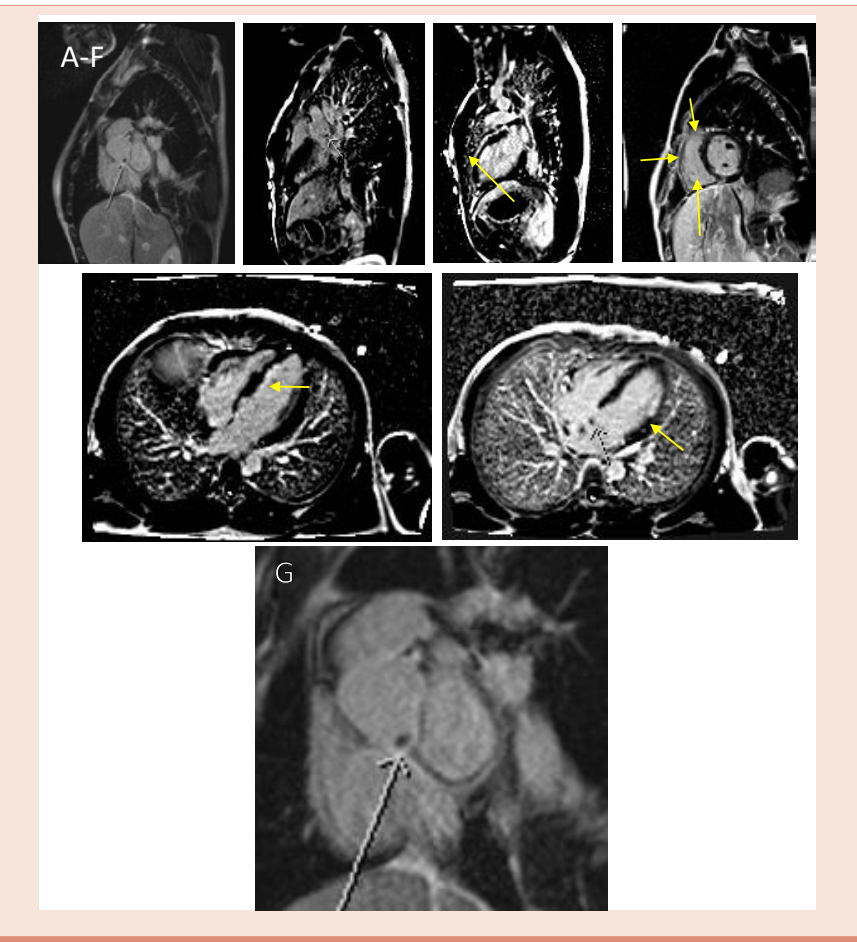

Figure 2: (A-F) Multiplanar postcontrast CMR imaging demonstrating thrombus on the aortic valve (white arrows). Subendocardial infarcts are also seen in the anterior, septal, inferior and lateral walls (yellow arrows). (G) Magnification of the aortic valve thrombus (white arrow).

the noncoronary sinus. Delayed enhanced imaging displayed large subendocardial infarcts in the RCA, PDA, left circumflex and first diagonal distributions (Figure 2C-F). Multiple areas of viable myocardium were also demonstrated. The ventricles were mildly dilated and systolic function was low normal.

Blood testing was positive for anticardiolipin antibodies (aCL) and the patient was started on heparin therapy. The patient underwent computed tomography of the abdomen to evaluate his elevated liver function tests. Budd-Chiari Syndrome with nonocclusive portal vein, superior mesenteric vein, and splenic vein thrombosis were identified. Additionally, inferior vena cava and right external iliac vein thrombosis, and bilateral renal infarcts were observed.
Coronary angiography was repeated prior to surgery demonstrating nonocclusive disease in the left coronary artery and $80-90 \%$ stenosis in the distal right coronary artery.

Surgery was performed the next day to bypass the RCA stenosis and remove the aortic valve thrombus (Figure $2 \mathrm{G}$ ). The right internal mammary artery was skeletonized and used as an in situ graft to the posterior descending artery and embolus was removed from the proximal portion of the right coronary artery. The aortic valve thrombus was also removed and was noted to be completely smooth and round with no stalk. Histopathologic examination of the aortic thrombus showed a fibrinous material infiltrated by histiocytes and neutrophils, consistent with an organizing thrombus. The patient was treated with heparin postoperatively and discharged with lifelong anti-coagulant treatment with Xarelto (rivaroxaban) $20 \mathrm{mg}$ orally once a day.

\section{Discussion}

There have been a few cases of myocardial infarction occurring in patients with APS published in the medical literature ${ }^{6}$. However, this is the first documented instance of an aortic valve thrombus identified on CMR in a patient with APS causing coronary artery embolic disease.

This finding is extremely rare and its low frequency of reporting in the medical literature may be due to one of two reasons. It was shown in our patient that conventional angiography and TTE were unable to detect the aortic valve thrombus. Only CMR was able to detect this abnormality. It may be that aortic valve thrombosis was not identified in some of the previously published antiphospholipid patients because they are only evaluated with coronary angiography and TTE. The other possibility is that our case is indeed a truly rare complication of APS.

\section{Conclusion}

Patients with antiphospholipid syndrome are at risk for venous and arterial thrombosis. We report a case of a young patient presenting with chest pain found to have coronary embolic disease caused by aortic valve thrombosis related to antiphospholipid syndrome. Accurate diagnosis was made using a combination of conventional coronary angiography and cardiac MR imaging. 


\section{References}

1. Miyakis S, Lockshin MD, Atsumi T, Branch DW, Brey RL, et al. (2006) International consensus statement on an update of the classification criteria for definite antiphospholipid syndrome (APS). J Thromb Haemost 4: 295-306.

2. Levine JS, Branch DW, Rauch J (2002) The antiphospholipid syndrome. N Engl J Med 346: 752-763

3. Petri M (2000) Epidemiology of the antiphospholipid antibody syndrome. J Autoimmun 15: 145-151.

4. Long BR, Leya F (2008) The role of antiphospholipid syndrome in cardiovascular disease. Hematol Oncol Clin North Am 22: 79-94.
5. Bulckaen HG, Puisieux FL, Bulckaen ED, Di Pompeo C, Bouillanne OM, et al. (2003) Antiphospholipid antibodies and the risk of thromboembolic events in valvular heart disease. Mayo Clin Proc 78: 294-298.

6. Cervera R, Piette JC, Font J, Khamashta MA, Shoenfeld Y, et al. (2002) Antiphospholipid syndrome: clinical and immunologic manifestations and patterns of disease expression in a cohort of 1,000 patients. Arthritis Rheum46: 1019-1027.

7. Shibata T, Kawakami S, Noguchi T, Tanaka T, Asaumi Y, et al. (2015) Prevalence, Clinical Features, and Prognosis of Acute Myocardial Infarction Attributable to Coronary Artery Embolism. Circulation 132: 241-250. 